\title{
LA DIPLOMACIA PARLAMENTARIA: EL PAPEL DE LOS PARLAMENTOS EN EL MUNDO
}

\author{
Stelios STAVRIDIS*
}

SUMARIO: 1 . INTRODUCCIÓN.-2. DIPLOMACIA PARLAMENTARIA: ¿OXÍMORON O REALIDAD?-2.1. De narrar una práctica al interés académico por esa práctica._-2.2. «Pre-historia» de la diplomacia parlamentaria.-2.3. Medios e instrumentos parlamentarios para la acción internacional.-2.4. Hacia una conceptualización de la diplomacia parlamentaria: la aportación académica.-3. EJEMPLOS DE DIPLOMACIA PARLAMENTARIA: CARACTERÍSTICAS Y FUNCIONES.-3.1. Flexibilidad/informalidad.-3.2. Complementariedad o alternativa.-3.3. Contribución a resolución de conflictos.-3.4. Tribunas morales.-4. LAS INSTITUCIONES PARLAMENTARIAS INTERNACIONALES (IPI) COMO DIPLOMACIA PARLAMENTARIA.-5. CONCLUSIONES.

\section{INTRODUCCIÓN}

1. En el sistema internacional actual, los Estados continúan siendo sus actores más importantes. La diplomacia — definida como «diálogo entre Estados independientes» $-{ }^{1}$ sigue dominada por gobiernos, ministerios, embajadas, tanto a nivel bilateral como multilateral. Sin embargo, en los últimos años, al papel tradicional de un diplomático (comunicación, reportaje, análisis de políticas, negociación y representación —se deja por el momento intencionalmente de lado el rol de defender los intereses nacionales-), se han añadido nuevas tareas, en gran parte como resultado de la globalización: eco-

* Dr. Stelios Stavridis es Investigador Senior ARAID en la Universidad de Zaragoza (dr.stelios. stavridis@gmail.com). Este texto se basa en una ponencia presentada durante la Jornada sobre Los Parlamentos en las relaciones internacionales organizada por la Fundación Manuel Giménez Abad de Estudios Parlamentarios y del Estado Autonómico y el Real Instituto Elcano de Estudios Internacionales y Estratégicos (Madrid) celebrada el 10 de mayo de 2018 en las Cortes de Aragón en Zaragoza. El autor agradece la oportunidad de presentar su trabajo a los organizadores del evento y, en particular, a José Tudela Aranda, Ignacio Molina, Mario Koëlling y José Sánchez Medalón. Extiende su agradecimiento a los tres evaluadores anónimos de REDI por sus comentarios y sugerencias constructivos sobre una versión posterior de esa presentación. Las traducciones al español en el texto son del autor. Todas las páginas web de referencia han sido consultadas por última vez el 29 de noviembre de 2018.

1 Watson, A., Diplomacy - The Dialogue Between States, Londres, Methuen, 1982. 
nomía, finanzas, terrorismo, cambio climático, migraciones, etc. También es posible hablar ahora de una diplomacia pública, un cambio que representa «en cierto sentido una metáfora de la democratización de la diplomacia, con múltiples actores que desempeñan un papel en lo que alguna vez fue un área restringida a unos pocos» ${ }^{2}$, y también de la pujanza del "poder blando» ${ }^{3}$.

2. El resultado de este mundo nuevo ha sido una proliferación de distintos actores internacionales ${ }^{4} \mathrm{y}$ de nuevas formas de diplomacia, con la adición de adjetivos a su conceptualización y práctica: por ejemplo, diplomacia económica ${ }^{5}$, cultural $^{6}$, de la defensa ${ }^{7}$, de las celebridades ${ }^{8}$, deportiva ${ }^{9}$, gastronómica $^{10}$, científica ${ }^{11}$, humanitaria ${ }^{12}$, paradiplomacia de las regiones y ciuda$\operatorname{des}^{13}, \mathrm{y}$-el tema de este artículo- diplomacia parlamentaria ${ }^{14}$. Todos estos procesos han sido posibles gracias a una multitud de avances tecnológicos y a la aparición y sofisticación continua de nuevos medios de comunicación.

\footnotetext{
2 Melissen, J., Beyond the New Public Diplomacy, Clingendael Paper No. 3, Netherlands Institute of International Relations «Clingendael», La Haya, 2011, p. 2.

3 Nye, J., Soft Power: Means to Success in World Politics, Nueva York, Public Affairs, 2004.

4 Reinalda, B. (ed.), The Ashgate Research Companion to Non-State Actors, Farnham, Ashgate, 2011.

5 Bayne, N. y Woolcock, S., The New Economic Diplomacy Decision-Making and Negotiation in International Economic Relations, Farnham, Ashgate, 2011.

6 KITsou, S., "The Power of Culture in Diplomacy: The Case of U.S. Cultural Diplomacy in France and Germany», Exchange, vol. 2, 2013, núm. 1, disponible en https://surface.syr.edu/exchange/vol2/ iss $1 / 3 /$.

7 Cheyre, J.-E., «Defence Diplomacy», en Cooper, A. F., Heine, J. y Thakur, R. (eds.), The Oxford Handbook of Modern Diplomacy, Oxford, OUP, 2013.

8 Huliaras, A. y TzIFAKIS, N., "Celebrity Activism in International Relations: In Search of a Framework for Analysis», Global Society, vol. 24, 2010, núm. 2, pp. 255-274.

9 The Hague Journal of Diplomacy, vol. 8, Special Issue on «Sports Diplomacy», 2013.

10 Lusa, D. y JAKESEvic, R., "The Role of Food in Diplomacy: Communicating and "Winning Hearts and Minds" Through Food», Medijske Studije/Media Studies, vol. 8, 2017, núm. 16, pp. 99-119.

11 Véase la revista Science and Diplomacy que acaba de cumplir su primera década publicada por el Center for Science Diplomacy de la American Association for the Advancement of Science, disponible en https://www.aaas.org/program/center-science-diplomacy.

12 RÉGNIER, Ph., "The emerging concept of humanitarian diplomacy: identification of a community of practice and prospects for international recognition", International Review of the Red Cross, vol. 93, 2011, núm. 884, pp. 1211-1237. Sobre la diplomacia humanitaria de España véase el portal dedicado al tema: "La Oficina de Acción Humanitaria realiza también una labor de sensibilización y seguimiento desde una perspectiva humanitaria en los debates internacionales y en los principales foros en los que España es parte», disponible en http://www.aecid.es/ES/ah/que/ diplomacia.

13 WolfF, S., «Paradiplomacy. Scope, Opportunities and Challenges», The SAIS Europe Journal of Global Affairs, vol. 10, 2007, pp. 141-150; PASQUIER, R., "Quand le local rencontre le global: contours et enjeux de l'action internationale des collectivités territoriales", Revue française d'administration publique, 2012, núm. 141, pp. 167-182; SKOUTARIs, N., «Comparing the Subnational Constitutional Space of the European Sub-State Entities in the Area of Foreign Affairs», Perspectives on Federalism, vol. 4, 2012, núm. 12, pp. 239-268. Véase también en algunos casos como los de Quebec, Escocia o Cataluña, la protodiplomacia. La protodiplomacia se relaciona con la búsqueda de conseguir un Estado independiente por parte de un pueblo o de un ente subestatal (McHugH, J. T., «Paradiplomacy, protodiplomacy and the foreign policy aspirations of Quebec and other Canadian provinces», Canadian Foreign Policy Journal, vol. 21, 2015, núm. 3, pp. 238-256).

14 La publicación más reciente sobre el tema es Stavridis, S. y JANCIC, D. (eds.), Parliamentary Diplomacy in European and Global Governance, Diplomatic Studies Series, Leiden/Boston, Brill Nijhoff, 2017.
} 
3. Brevemente, las instituciones parlamentarias se involucran en asuntos internacionales de tres maneras principales ${ }^{15}$ : a través del control e influencia en las políticas exteriores de los gobiernos nacionales; realizando unas actividades y acciones internacionales, conocidas como diplomacia parlamentaria; y estableciendo y empoderando los parlamentos como órganos representativos de organizaciones regionales o globales, principalmente a través de instituciones parlamentarias internacionales (IPI).

4. Ejemplos en temas internacionales abundan: el día después del anuncio por parte del presidente Donald Trump de la retirada de Estados Unidos del acuerdo internacional sobre el nuclear iraní, parlamentarios de este país quemaron la bandera estadounidense dentro del recinto legislativo en Teherán con gritos de «iMuerte a Estados Unidos!» ${ }^{16}$. En el verano de 2017, diputados alemanes no pudieron visitar a soldados de su país desplegados en la base turca de Incirlik y, como resultado, dichas tropas fueron trasladadas a Jordania - la razón de la interdicción turca a esta visita fue la culminación de meses de tensiones entre los dos países, resultado de la aprobación en el Bundestag de una resolución condenando el genocidio armenio- ${ }^{17}$. En octubre de 2017, Marruecos impidió, y no por primera vez, la entrada de eurodiputados, pertenecientes al Intergrupo del Parlamento Europeo (PE) sobre el Sáhara Occidental, a estos territorios ocupados. Ni siquiera pudieron bajar del avión en el que viajaban ${ }^{18}$.

5. Pero, ¿son todos ejemplos de la existencia de una «diplomacia parlamentaria»? ¿Qué significa este término? ¿Representa un espejismo basado sobre un oxímoron, o, al contrario, un fenómeno reciente que se está consolidando a nivel mundial?

Este artículo argumenta que, basándose en datos empíricos, existe en todo el mundo una esfera parlamentaria multi-capas, multi-niveles, y multi-actores que, hasta cierto punto, se refuerzan mutuamente: es decir un contexto amplio de parlamentarización del sistema internacional ${ }^{19}$, facilitado por una cooperación interparlamentaria cada vez más asentada y sofisticada —en particular, pero no solo-, en Europa ${ }^{20}$. Este estudio se centrará en el caso

15 Malamud, A. y Stavridis, S., «Parliaments and Parliamentarians as International Actors», en Reinalda, B. (ed.), The Ashgate Research Companion to Non-State Actors, Farnham, Ashgate, 2011, pp. 101-115, esp. p. 101.

16 www.20minutos.es, 9 de mayo de 2018.

17 www.rtve.es/noticias/20170607/alemania-retira-tropas-base-turca-incirlik-tras-meses-tensionesgobierno-erdogan/1561530.shtml, 7 de junio de 2017.

18 www.publico.es/internacional/marruecos-impide-entrada-cinco-eurodiputados-territorios-ocupados-sahara-occidental.html, 27 de octubre de 2017.

19 CRUM, B. y Fossum, J. E. (eds.), Practices of interparliamentary coordination in international politics, Colchester, ECPR Press, 2013; Costa, O., Dri, C. y STAVRIDIs, S. (eds.), Parliamentary Dimensions of Regionalization and Globalization, Basingstoke, Palgrave Macmillan, 2013; JANCIC, D., "Globalizing Representative Democracy: The Emergence of Multilayered International Parliamentarism», Hastings International and Comparative Law Review, vol. 38, 2015, núm. 2, pp. 197-242.

${ }^{20}$ Véase Lupo, N. y FASONE, C. (eds.), Interparliamentary Cooperation in the Composite European Constitution, Oxford y Portland Oregon, Hart Publishing, 2016; Griglio, E. y STaVridis, S. (eds.), Special Issue on "Joint scrutiny of EU policies: the contribution of interparliamentary cooperation», 
de la diplomacia parlamentaria per se, y de las IPI desde la perspectiva de la aportación de estas últimas a la diplomacia parlamentaria.

\section{DIPLOMACIA PARLAMENTARIA: ¿OXÍMORON O REALIDAD?}

\subsection{De narrar una práctica al interés académico por esa práctica}

6. Esta sección explica cómo hemos pasado de una aproximación descriptiva por parte de practitioners a un principio de análisis en el mundo académico de lo que representa la diplomacia parlamentaria como fenómeno global. De algo fundamentalmente descriptivo como resultado de la propia experiencia de los parlamentarios y funcionarios parlamentarios que practican la diplomacia parlamentaria diariamente, a un esbozo de estudio académico e, incluso, a una consolidación de este interés del mundo universitario. Falta investigación para llegar a definir criterios de evaluación de la diplomacia parlamentaria, de forma que es algo que no se ha conseguido completamente hasta la fecha.

7. ¿Es la «diplomacia parlamentaria» solo un oxímoron aparente? Un oxímoron es una figura retórica de pensamiento que consiste en complementar una palabra con otra que tiene un significado contradictorio u opuesto. Es también una reacción muy común cuando alguien menciona la diplomacia parlamentaria. Es además correcto indicar que durante años había un déficit académico preocupante sobre el tema, algo que ha cambiado hace poco tiempo. Pero en realidad, no es un oxímoron, sino todo lo contrario. Como veremos, la diplomacia parlamentaria no solo representa una práctica desarrollada en todo el mundo, sino que se puede apreciar una consolidación de este fenómeno tanto geográficamente como sectorialmente, después de la Segunda Guerra Mundial, consecuencia de los procesos de integración regional, y especialmente desde el final de la Guerra Fría, con una continua proliferación de IPI de todo tipo. Aunque se puede argumentar que existe una crisis de la democracia representativa con lo que esto conlleva para los Parlamentos, no se puede discutir que nuevas formas de democracia (basadas a menudo sobre avances tecnológicos) han mostrado igualmente sus limitaciones y riesgos. En cuanto a la llamada «democracia iliberal»—o mejor dicho, «autocracia electoral»—- ${ }^{21}$, estamos llegando a entender el oxímoron de esta conceptualización con las experiencias reales en algunos países del Este de Europa, Turquía o Rusia. La democracia siendo, en las famosas palabras de Winston Churchill, «el peor de todos los sistemas políticos, con excepción de todos los sistemas políticos restantes». Los

\footnotetext{
Perspectives on Federalism, vol. 10, 2018, núm. 3, pp. i-xviii y pp. 1-21, disponible en http://www.onfederalism.eu/attachments/004_Volume\%2010\%20-\%20issue\%203\%20-\%202018.pdf.

${ }^{21}$ En las palabras del profesor de Derecho europeo de la Universidad de Leiden y filósofo político van MiddelaAR, L., "La Hongrie est devenue une autocratie électorale», Le Monde, 23-24 de septiembre de 2018 .
} 
parlamentos y los parlamentarios continúan situados en el «corazón» de los sistemas democráticos.

\section{2. «Pre-historia» de la diplomacia parlamentaria}

8. Al principio, el término «diplomacia parlamentaria» fue utilizado de una manera muy específica: refiriéndose a la llamada «diplomacia de la conferencia ${ }^{22}$, es decir, debatir públicamente dentro de un foro internacional con la intención en particular de intentar solucionar conflictos, promover la paz, en definitiva, la justicia internacional. Los participantes podían ser parlamentarios pero, en general, eran representantes de los Estados. En el siglo Xx este tipo de diplomacia culminó con la creación de la Sociedad de Naciones (SDN) y más tarde la de la Organización de las Naciones Unidas $(\mathrm{ONU})^{23}$.

9. En consecuencia, el término diplomacia parlamentaria no se refería a parlamentarios sino a un procedimiento, o un método, para intentar prevenir o solucionar conflictos entre las entidades que forman el sistema internacional: «Una combinación sui generis de parlamentarismo y de diplomacia», como escribía, en 1997, César Sepúlveda ${ }^{24}$. En definitiva, una nueva manera abierta y pública de hacer diplomacia, pero que existía desde los tiempos antiguos. "La diplomacia de la conferencia tiene sus antecedentes en el Mediterráneo oriental en el siglo IV a. C., cuando las ciudades-estado griegas y Persia convocaron ocho congresos políticos internacionales y establecieron un status quo territorial mutuamente garantizado junto con reglas de conducta acordadas para regular los asuntos internacionales ${ }^{25}$.

10. A finales del siglo XIX los parlamentarios crearon la Unión Inter-Parlamentaria (UIP) para intentar promover tanto la paz como la justicia internacional (fundada en 1889 por dos parlamentarios, el francés Frédéric Passy y el británico William Randal Cremer). Es decir que desde 1889 existe una «organización mundial de los parlamentos nacionales. Es el centro del diálogo y la diplomacia parlamentaria entre legisladores de todos los sistemas políticos del mundo. [...] Sus objetivos son: promover la paz y la seguridad

${ }^{22}$ Rosenbaum, N., "Cyprus and the United Nations: An Appreciation of Parliamentary Diplomacy", The Canadian Journal of Economics and Political Science / Revue canadienne d' Économie et de Science politique, vol. 33, 1967, núm. 2, pp. 218-231; LATEEF, N., «Parliamentary Diplomacy and the North-South Dialogue», Georgia Journal of International and Comparative Law, vol. 11, 1981, núm. 1, pp. 1-44; RitTBERgER, V., «Global Conference Diplomacy and International Policy-Making: The Case of UN-Sponsored World Conferences», European Journal of Political Research, 1983, pp. 167-182.

${ }_{23}$ Véase RusK, D., "Parliamentary Diplomacy-Debate vs. Negotiation», World Affairs Interpreter, vol. 26, 1955, núm. 2, pp. 121-138.

${ }^{24}$ Citado en CALDERÓN PATiÑo, J.-P., Los retos de la diplomacia clásica y la función de la diplomacia parlamentaria, IBERAM, 2003, p. 9. Véase también SEPÚLVEDA, C., «La diplomacia parlamentaria y la formación de un nuevo orden jurídico internacional», Estudios de Derecho Internacional en Homenaje al Profesor Miaja de la Muela, t. II, Madrid, Tecnos, 1979, pp. 773-791.

${ }^{25}$ Cooper, A. F., Heine, J. y Thakur, R., «Introduction: The Challenges of 21st-Century Diplomacy», en Cooper, A. F., Heine, J. y ThaKur, R. (eds.), op. cit., nota 7, pp. 1-35, esp. p. 21. 
mediante el diálogo político y la diplomacia parlamentaria, fortalecer la democracia y el respeto a los derechos humanos y contribuir al desarrollo de instituciones legislativas representativas y eficaces, como así también al desarrollo económico y social de los países» ${ }^{26}$.

11. Hoy en día, en 2018 , la UIP tiene 178 parlamentos miembros y 12 miembros asociados ${ }^{27}$. Pero como ya ha sido mencionado, no solo se ocupa de diplomacia parlamentaria: su objetivo específico es la promoción de la democracia parlamentaria, algo más típico de la cooperación técnica entre parlamentos, es decir, una función más tradicional de los parlamentos en sus relaciones internacionales. Sin olvidar, por supuesto, que existe un vínculo entre democratización y diplomacia parlamentaria, y «al participar en foros internacionales, los parlamentarios obtienen acceso a la información y el conocimiento global que pueden contribuir a su trabajo al nivel nacional» ${ }^{28}$. En otras palabras, lo que Lorinc Redei ha calificado como «el refuerzo mutuo de la supervisión parlamentaria y de la diplomacia parlamentaria» ${ }^{29}$. En ese sentido, Giovanna Bono también muestra cómo en las dos primeras operaciones militares dirigidas por la Unión Europea (UE) (EUFOR Concordia y EUFOR Artemis), ambas iniciadas en 2003, diputados italianos encontraron la información que su propio gobierno se negaba a proporcionarles contactando directamente a sus contrapartes en el Reino Unido, ya que ambos países estaban involucrados en las operaciones ${ }^{30}$. Y como también el otro objetivo importante de la UIP es la promoción de la justicia internacional con su apoyo a la creación de tribunales internacionales y a la mediación en conflictos, se puede resumir su papel como una contribución parlamentaria a la pacificación y la democratización del sistema internacional.

\subsection{Medios e instrumentos parlamentarios para la acción internacional}

12. Muchos de los medios e instrumentos parlamentarios que existen en general se utilizan también en relaciones exteriores, incluso en el caso de la diplomacia parlamentaria. Hay también herramientas particularmente adaptadas a la acción exterior de los parlamentos y otras asambleas parla-

\footnotetext{
26 VÁzouez, R., "La diplomacia parlamentaria», Revista Relaciones Internacionales, vol. 21, UNLP, 2001, pp. 261-271, esp. p. 261.

27 https://www.ipu.org/about-us/members.

28 Palabras del diputado finlandés Kimno Kiljunen en 2004 citado en SABIC, Z., «Building democratic and responsible global governance: the role of international parliamentary institutions», Parliamentary Affairs, vol. 61, 2008, núm. 2, pp. 255-271, esp. p. 268.

29 REDEI, L., «The European Parliament as a diplomatic precedent setter: the case of parliamentary relations with Kosovo", en STAVridis, S. e IrRera, D. (eds.), The European Parliament and its International Relations, Londres, Routledge, 2015, pp. 272-285.

30 Bono, G., «National Parliaments and EU External Military Operations: Is there any Parliamentary Control?», European Security, vol. 14, 2007, núm. 2, pp. 203-229.
} 
mentarias. En la primera categoría se incluyen la ratificación de acuerdos internacionales, debates en plenarios, preguntas escritas u orales, informes, comparecencias, y por supuesto la aprobación presupuestaria. En la segunda, comisiones especializadas (exterior, seguridad, defensa, comercio exterior, ayuda al desarrollo), delegaciones (tanto bilaterales como multilaterales) con países o regiones terceras, visitas de parlamentarios en estos Estados $\mathrm{u}$ organizaciones internacionales y recepción de homólogos, intercambios entre partidos políticos (o «familias políticas»), y finalmente grupos de amistad. En todos estos casos hay que hacer hincapié sobre la importancia de los vínculos personales, una dimensión que también implica habilidades lingüísticas, sociales, etc. Solo para poner unos ejemplos, en el Parlamento Europeo (PE), en la legislatura actual, hay 44 delegaciones con terceros países y otras regiones, y en el Parlamento griego existen en estos momentos unos 75 grupos de amistad.

13. Tradicionalmente los parlamentos han sido muy activos en la cooperación técnica interparlamentaria, bilateral o multilateral, implicando numerosos intercambios de diputados y de funcionarios parlamentarios (buenas prácticas) que incluyen una importante dimensión de «socialización» y de «democratización». Por ejemplo, el «Parlamento [de Canadá] organiza un programa de estudio para funcionarios parlamentarios varias veces al año. Este programa de desarrollo profesional ofrece al personal parlamentario senior de todo el mundo la oportunidad de intercambiar puntos de vista y mejores prácticas en materia de procedimientos, cuestiones administrativas y temas de investigación» ${ }^{31}$. En el caso español, en 2008, la letrada de las Cortes Generales, Piedad García-Escudero Márquez ${ }^{32}$, listaba las siguientes actividades: el "Aula Parlamentaria», seminarios especiales, actividades conjuntas con la Fundación Internacional y para Iberoamérica de administración y políticas públicas, y con el centro en España del Banco Mundial ${ }^{33}$.

14. Además, existe una significativa participación parlamentaria en misiones de observación electoral. Así, Laura Feliu y Francesc Serra señalan que «desde 1999, el PE ha enviado más de 100 misiones de observación a 29 países africanos, 18 en Europa del Este y la CEI, 10 en América Latina, 11 en Asia y Oceanía» ${ }^{34}$. Finalmente, no se debe despreciar, de hecho todo lo contrario, el rol de «correa de transmisión entre ejecutivo y sociedad civil» que un ente parlamentario desempeña en particular en países democráticos,

31 Mychajlyszyn, N., Parliamentary Diplomacy, Canadian Parliamentarians and the World, 8 de diciembre de 2015, disponible en http://hillnotes.ca/2015/12/08/parliamentary-diplomacy-canadian-parliamentarians-and-the-world/.

32 También catedrática de Derecho constitucional en la Universidad Complutense de Madrid.

33 García-Escudero Márouez, P., «Diplomacia y Cooperación Parlamentarias: las Cortes Generales», Asamblea-Revista Parlamentaria de la Asamblea de Madrid, diciembre de 2008, núm. 19, pp. 3-32; ejemplos pp. 26-30.

34 Feliu, L. y Serra, F., "The European Union as a "normative power" and the normative voice of the European Parliament», en STAVridis, S. e Irrera, D. (eds.), op. cit., nota 29, pp. 17-34, esp. p. 28. 
ni el de participación por parte de parlamentarios en debates «de opinión» en la prensa ${ }^{35} \mathrm{y}$ otros medios de comunicación, incluso medios sociales, o en actividades diversas, como coloquios o conferencias.

\subsection{Hacia una conceptualización de la diplomacia parlamentaria: la aportación académica}

15. El interés por algo más que la diplomacia de conferencia se produjo gracias a la ya indicada aparición de nuevas IPI a principios del siglo XX (Consejo nórdico y la Asociación Parlamentaria de la Commonwealth), con la cualitativa y cuantitativa proliferación de estas asambleas después de la Segunda Guerra Mundial (especialmente en Europa y en América Latina), y plasmado en una serie de artículos en la prensa u otras publicaciones por parlamentarios o diplomáticos ${ }^{36}$, muchos de tipo anecdótico pero también de testimonios más desarrollados, todos, es verdad, bastante descriptivos ${ }^{37}$. Hay también informes de las mismas asambleas parlamentarias como la de la Unión de Europa Occidental (UEO) en $2000^{38}$, y la organización de eventos sobre el tema como el promovido por el Senado francés en 2001 ${ }^{39}$; un fenómeno que ha continuado desde entonces, emprendido, a veces conjuntamente, con instituciones académicas o think tanks o ambos ${ }^{40}$.

16. Este proceso aporta una contribución interesante: la aparición de las primeras definiciones de la diplomacia parlamentaria pero, considerando el nivel poco avanzado de la investigación sobre este tema, solo son de índole descriptivo. De lejos la más citada es la de unos practitioners (sena-

35 Por ejemplo, en la actualidad sobre la cuestión nuclear en Corea del Norte, véase MENÉNDEZ DEL VALLE, E. (ex embajador y ex eurodiputado), «La paz en la península coreana ha de ser justa», El País, 27 de abril de 2018 .

36 Orozco Deza, M.-Á. (diputado mexicano), «Diplomacia parlamentaria», Revista Mexicana de Política Exterior, vols. 62-63, 2001, pp. 91-109; Trillo, F. (diputado español), "La diplomacia parlamentaria», El País, 2 de abril de 1997; Elorriaga Fernández, G. (senador español), "La diplomacia parlamentaria», Revista de las Cortes Generales, vol. 54, 2001, pp. 7-20.

37 Vázouez, R., op. cit., nota 26; Elorriaga, G., La Diplomacia Parlamentaria, Madrid, Imagine Ediciones, 2004.

38 Souncialupi, V., Parliamentary Diplomacy: The Role of International Assemblies, Report Submitted on Behalf of the Committee for Parliamentary and Public Relations, Assembly of Western European Union, Document A/1685 of June, Bruselas, 2000.

39 SÉnAT, La diplomatie parlementaire, Actes du Colloque, Paris, Sénat français, 2001. Véase también Parlamentary Centre, Parliamentary Diplomacy, Occasional Papers on Parliamentary Government 16, Ottawa, 2003.

40 Por ejemplo, Jornada sobre «Parlamento y Acción Exterior: la Diplomacia Parlamentaria», organizada por el Instituto de Derecho Parlamentario y la Facultad de Derecho de la Universidad Complutense de Madrid celebrada el 16 de diciembre de 2010 en Madrid; el Congreso sobre «Parliamentary diplomacy in the Mediterranean region», organizado por el Parlamento Helénico y el Instituto de Relaciones Económicas Internacionales, los días 26-27 de septiembre de 2013 en Atenas; o, la Mesa Redonda sobre «Parliamentary Diplomacy in the Mediterranean» organizada por la Universitat Autònoma de Barcelona/UAB, ARAID y la Universidad de Zaragoza el 12 de septiembre de 2017 en el CIDOB (Barcelona). 
dores de los Países Bajos) publicada en una revista dedicada al estudio de la diplomacia:

«La gama completa de actividades internacionales emprendidas por los parlamentarios para aumentar el entendimiento mutuo entre los países, ayudarse mutuamente en mejorar el control de los gobiernos y la representación de los ciudadanos y aumentar la legitimidad democrática de las instituciones intergubernamentales ${ }^{41}$.

17. Y otra muy similar, que se menciona menos y no ha aparecido en una revista académica pero que refleja el mismo sesgo descriptivo:

«La diplomacia parlamentaria comprende un gran número de actividades que incluyen: la conclusión de acuerdos de cooperación interparlamentaria para promover las relaciones entre países; la organización de encuentros y visitas institucionalizados y regulares entre parlamentarios, dirigidos tanto a la resolución de conflictos como al fomento de la confianza, así como al intercambio de conocimientos; el establecimiento de grupos de amistad y comités ad hoc; recibir y enviar delegaciones parlamentarias; y la participación en organismos y conferencias interparlamentarios multilaterales, entre los que destacan $\operatorname{los} \mathrm{IPI} \gg{ }^{42}$.

18. Otras definiciones descriptivas se han seguido produciendo a lo largo de los años, pero una más reciente e ilustrativa, desarrollada por un practitioner, es la siguiente:

«La totalidad de las actividades que lleva a cabo cada parlamento nacional, tanto como entidad colectiva como iniciativas de parlamentarios individuales, en sus propias relaciones con otros parlamentos nacionales o con los llamados parlamentos internacionales» ${ }^{43}$.

19. Todas estas definiciones han despertado el interés del mundo académico con la intención de ir más allá de listados detallados de las actividades internacionales parlamentarias. Esta nueva aproximación ${ }^{44}$ aborda el cómo y el por qué, sin conformase con solo describir la diplomacia parlamentaria como se había hecho hasta entonces. En el apartado siguiente se recogen ejemplos de estos avances.

41 Weisglas, F. y De Boer, G., «Parliamentary Diplomacy», The Hague Journal of Diplomacy, vol. 2, 2007, núm. 1, pp. 93-99, esp. pp. 93-94.

42 IPU, "Parliamentary Involvement in International Affairs», Report to II World Conference of Speakers of Parliaments, Nueva York, 7-9 de septiembre de 2005, p. 8.

43 Piazzi, S., Contribution by Ambassador Sergio Piazzi, Secretary General of the Parliamentary Assembly of the Mediterranean (PAM) on the occasion of the conference: South East European cooperation process parliamentary assembly - regional synergies, strategic cooperation and parliamentary diplomacy (Sofía, Bulgaria, 6 de noviembre de 2015); en la misma línea, véase también GARcía-EscudERo Márquez, P., op. cit., nota 33; Dirección de Relaciones Internacionales Parlamentarias, Diplomacia Parlamentaria de la Asamblea Nacional de Nicaragua, Managua, 2015.

${ }_{44}$ Véase, en particular, STAVRIDIS, S., "Parliamentary Diplomacy: Some Preliminary Findings», Jean Monnet Working Papers in Comparative and International Politics of the University of Catania, Catania, 2002, núm. 48; Beetham, D., Parliament and Democracy in the Twenty-first Century: A Guide to Good Practice, Interparliamentary Union, 2006. 


\section{EJEMPLOS DE DIPLOMACIA PARLAMENTARIA: CARACTERÍSTICAS Y FUNCIONES}

20. La literatura ha identificado una serie de características y funciones para la diplomacia parlamentaria como un fenómeno global ${ }^{45}$. Las publicaciones más recientes sobre el tema llegan a un conjunto de conclusiones preliminares sustentadas en una plétora de estudios de casos, llevados a cabo como parte de varios proyectos de investigación académica: se examinan 35 casos en todo el mundo (24 sobre las relaciones internacionales del Parlamento Europeo y otros 11 sobre diferentes asambleas parlamentarias, incluidas las nacionales), además de otras publicaciones previas, que incluyen más estudios de $\operatorname{casos}^{46}$.

21. Por tanto, el estudio de la diplomacia parlamentaria ha entrado en una nueva fase de análisis sistemático y exhaustivo, incluso de tipo comparativo. Las principales características atribuidas al rol, actividad e impacto internacionales de los parlamentarios en el mundo son: primero, que se trata de un proceso flexible y a menudo informal; segundo, que suponen niveles múltiples y capas múltiples, a menudo superpuestas (y que se refuerzan mutuamente) que van desde lo local a lo global pasando por lo nacional, lo regional y lo interregional; y, tercero, que involucran a múltiples actores, ya que los parlamentarios interactúan no solo con sus homólogos, sino también con otras personas y entidades en asuntos mundiales, tales como representantes estatales, actores de la sociedad civil, o de micro y macro regiones, etc. La literatura también ha identificado una pregunta crucial sobre si la diplomacia parlamentaria: ¿Es, y/o debería ser, complementaria o alternativa a la diplomacia estatal tradicional?

22. Lo que sigue ilustra algunos de estos puntos. No se pretende ofrecer un análisis completo de la diplomacia parlamentaria, sino simplemente abordar algunas cuestiones clave que este interés académico reciente ha identificado, en particular, con miras a futuras investigaciones.

\subsection{Flexibilidad/informalidad}

23. Los parlamentarios «son representantes de un pueblo, lo que tal vez les da más peso político que las ONG, pero al mismo tiempo sus acciones no necesariamente comprometen a un gobierno, lo que puede facilitarles el operar en situaciones particularmente delicadas ${ }^{47}$. Por tanto, durante o después de conflictos militares, los parlamentarios pueden actuar para mejorar

45 Lo que sigue se basa ampliamente en STAVRIDIs, S., «Conclusions: Parliamentary Diplomacy as a Global Phenomenon», en STAVRIDIS, S. y JANCIC, D., op. cit., nota 14, pp. 368-338.

46 Véanse los ensayos y artículos en Stavridis, S. y Gianniou, M. (eds.), Special issue on «Parliamentary Diplomacy in the Mediterranean», Mediterranean Quarterly: A Journal of Global Issues, vol. 27, 2016, núm. 4, pp. 2-148; y en STAVRIDIS, S. y JANCIC, D., op. cit., nota 14.

47 Weisglas, F. y DE Boer, G., op. cit., nota 41, p. 93, cursiva añadida. 
las relaciones entre los beligerantes antes de cualquier avance diplomático real. Esto puede lograrse a nivel individual, por ejemplo, el éxito del senador estadounidense Jesse Jackson en la liberación de prisioneros de guerra en Irak en 1991 o en Serbia en 1999. Y, de manera colectiva, puede conseguirse facilitando cambios en cuestiones fundamentales de política exterior de un Estado, como fue el caso con el reconocimiento del Estado de Israel por Grecia en $1990^{48}$, o las actuales presiones para el reconocimiento del Estado palestino, teniendo en cuenta la reciente avalancha de votos parlamentarios a favor de un Estado palestino ${ }^{49}$. Otro ejemplo es el hecho de que parlamentarios israelíes y palestinos sigan reuniéndose en las IPI incluso cuando hay crisis graves entre estas dos partes en la región ${ }^{50}$.

24. Franklin de Vrieze utiliza el ejemplo de Kosovo en el Proceso de Cooperación de Europa Sudoriental, y en particular en cómo la reconciliación regional representa también la «diplomacia parlamentaria en acción» ${ }^{51}$. Lo hace a través de dos ejemplos específicos: uno es la creación de la Secretaría de la Asamblea Parlamentaria de este Proceso, y el otro es la inclusión de Kosovo en su dimensión parlamentaria, a pesar de no ser reconocido como un Estado independiente por una serie de Estados, incluidos cinco miembros de la UE ${ }^{52}$. No obstante, el hecho de que Serbia no estuviera de acuerdo con la presencia kosovar no bloqueó su participación en la asamblea: un ejemplo práctico de la influencia parlamentaria sobre la diplomacia tradicional. Será interesante ver cómo en el futuro se desarrolla este ejemplo de diplomacia parlamentaria, en particular en vista de la presidencia kosovar de dicha IPI prevista para 2019-2020.

\subsection{Complementariedad o alternativa}

25. Este es un tema clave de la diplomacia parlamentaria que la literatura también ha planteado después de tal identificación por parte de los propios

48 "A First in Greek-Israeli Relations: Greek Parliamentary Delegation to Visit Israel Next May», Jewish Telegraphic Agency, 30 de diciembre de 1986, disponible en http://www.jta.org/1986/12/30/ archive/a-first-in-greek-israeli-relations-greek-parliamentary-delegationto-visit-israel-next-may.

49 Oliveira Martins, B., "A Sense of Urgency”: The EU, EU Member States and the Recognition of the Palestinian State», Mediterranean Politics, vol. 20, 2015, núm. 2, pp. 281-287; STAVRIDIS, S., Gianniou, M. y Cofelice, A., «EU national Parliaments and the recognition of Palestine: "Really" breaking new ground or "just" adding further support?», Comillas Journal of International Relations, 2016, núm. 6, pp. 40-60, disponible en http: //revistas.upcomillas.es/index.php/internationalrelations/article/ view/6961/6777.

50 Véase el ejemplo citado por Jordi Pedret, diputado español, durante la crisis de Gaza en 2008: PEDRET, J., «La Asamblea euromediterránea y la protección de derechos humanos», en GARZón CLARIANA, G. (ed.), La Asamblea Euromediterránea - The Euro-Mediterranean Assembly - L'assemblée euro-méditerranéenne, Madrid-Barcelona-Buenos Aires, Marcial Pons, 2011, pp. 121-135, esp. p. 134.

51 De VRIEze, F., «The South-East European Cooperation Process and its New Parliamentary Assembly: Regional Dialogue in Action», en STAVRIDIS, S. y JANCIC, D., op. cit., nota 14, pp. 174-192.

${ }^{52}$ Para el caso español, véase «España rebaja al máximo su presencia en una cita de la UE con Kosovo», El País, 16 de mayo de 2018. El presidente del Gobierno español fue el único que no salió en la foto oficial final del evento celebrado en Sofía bajo Presidencia búlgara. 
profesionales en publicaciones previas. Hay dos tendencias fácilmente identificables: por un lado, aquellos que reclaman que no solo es imposible sino indeseable utilizar la diplomacia parlamentaria para socavar la diplomacia estatal $^{53} \mathrm{y}$, por otro lado, aquellos que afirman que, como un alto asesor del presidente del Senado francés lo ha expresado ${ }^{54}$ : «Hacemos cosas que el Ejecutivo no puede hacer; de lo contrario, ¿para qué servirían nuestras actividades internacionales? ${ }^{55}$. Sin entrar en los detalles de este importante debate, el punto de vista preponderante es el que afirma que, como lo ha declarado la ex presidenta del Comité de Asuntos Exteriores de la Asamblea Nacional francesa, y ex ministra y ex eurodiputada Elisabeth Guigou: «Ciertamente no es deseable [que el Parlamento] ejerza un veto o desarrolle una diplomacia paralela. Por otro lado, sería muy útil que los expertos parlamentarios desarrollen su actividad internacional apoyando la diplomacia del ejecutivo mediante un diálogo y una consulta estrechos con este» ${ }^{56}$.

26. Hay numerosos ejemplos de diplomacia parlamentaria que se consideran no solo como una interferencia, sino también como una complicación para las relaciones tradicionales de Estado a Estado. Algunos ejemplos han sido la visita de la presidenta del Comité de Asuntos Exteriores del Congreso de los Estados Unidos Nancy Pelosi a Siria en 2007, a pesar de que la política oficial de Estados Unidos era contraria a tales contactos ${ }^{57}$, o las visitas continuas de parlamentarios franceses a Damasco en medio de las masacres continuas de civiles de la fracasada «Primavera Árabe» en ese país ${ }^{58}$. También, cabe mencionar la invitación a principios de 2015 al primer ministro de Israel por parte de congresistas republicanos ${ }^{59}$, desafiando las advertencias de la Casa Blanca entonces bajo presidencia demócrata (Barack Obama), algo que llevó a varios observadores a hablar de un acto «inconstitucional» ${ }^{60}$.

53 Trillo, F., op. cit., nota 36; García-Escudero Márouez, P., op. cit., nota 33, p. 6: «Complementa a la diplomacia clásica del ejecutivo, con la que debe cooperar, sin olvidar que al ejecutivo le corresponde la dirección de la política exterior (así, en el art. 97 de la Constitución española). Lo que es correcto desde el punto de vista del Derecho constitucional en este caso, pero extremamente reductor desde la perspectiva más amplia de las Relaciones Internacionales».

54 Martin-LaLande, N., «L'affirmation opportune d'une diplomatie parlementaire alternative», 5 de abril de 2007, disponible en http://www.dandurand.uqam.ca.

55 Entrevista informal con el autor en París, 16 de febrero de 2016.

56 Guigou, E., "Article pour la revue de l'ENA», Assemblée Nationale, Commission des Affaires étrangères, París, 19 de febrero de 2015.

57 Martin-Lalande, N., op. cit., nota 54.

58 «Syrie: Quatre élus français ont rencontré Bachar Al-Assad à Damas», Le Monde, 25 de febrero de 2015; "Le bureau de l'Assemblée nationale maintien l'agrément du groupe d'amitié France-Syrie», Le Monde, 19 de marzo de 2015; «Trois députés de la majorité prévoient de se rendre en Syrie fin septembre», Le Monde, 21 de septiembre de 2015.

59 BASSETS, M., «Netanyahu augura que el pacto con Irán lleva a una "pesadilla nuclear". El primer ministro israelí intenta frenar en Washington el acuerdo de Obama con Irán», El País, 4 de marzo de 2015.

60 CoBBs, E. A., «Why Boehner's invite to Netanyahu is unconstitutional», 2 de febrero de 2015, disponible en http://blogs.reuters.com/great-debate/2015/03/01/netanyahu-invite-is-a-symptom-of-boehnersgrudge-match-against-the-u-s-constitution/. 


\subsection{Contribución a resolución de conflictos}

27. En un estudio que hizo David Beetham para la UIP se mencionaban hasta una decena de ejemplos donde parlamentarios habían intentado facilitar soluciones a crisis internacionales y a estabilizar regiones y países en el mundo desde Oriente Medio, en particular Iraq e Irán (sobre la región incluso Afganistán y Palestina); Marruecos (sobre terrorismo), hasta Cabo Verde y Mozambique (sus presidentes actuaron en nombre de los presidentes de los parlamentos lusófonos sobre la crisis en Guinea-Bissau), Armenia, Azerbaiyán, Georgia y Francia (sobre Nagorno-Karabakh) ${ }^{61}$. Un estudio sobre la diplomacia parlamentaria canadiense ofrece una lista de los conflictos internacionales donde esta última ha intervenido: Vietnam, Sierra Leona, Kosovo, Sudán, y Corea del Norte ${ }^{62}$.

28. Resulta interesante citar dos ejemplos más, uno en África y el otro en Europa. El primero es el «Foro Amani», un foro parlamentario para impulsar la paz en los Grandes Lagos creado en 1999 en Kampala por los parlamentos nacionales de Burundi, República Democrática del Congo, Kenia, Tanzania, Uganda, Gambia más la Asamblea Legislativa del África Oriental. Al final reunió a 800 parlamentarios que produjeron informes, organizaron misiones de información, congresos internacionales, y varias comparecencias públicas. De este modo contribuyó al éxito de las negociaciones internacionales de paz. A medida que la región se ha vuelto más pacífica, el Foro perdió su razón de ser original y fue desmantelado en $2012^{63}$.

29. El segundo ejemplo es la «Misión Cox-Kwasniewski» (2012-2013), una misión conjunta en Ucrania del ex presidente del Parlamento Europeo Pat Cox y del ex presidente de Polonia Aleksander Kwasniewski con el objetivo inicial de mejorar las condiciones de presos políticos, y su posible liberación ${ }^{64}$. Se extendió más allá tanto en el tiempo como en sus metas. Contribuyó a rebajar tensiones entre Ucrania y Rusia, facilitando un diálogo entre ucranianos, con el objetivo de abrir la puerta a una posible consolidación de la democracia en este país. Finalmente, la misión permitió la liberación de varios presos políticos y la mejora en las condiciones de detención de otros, además de consolidar la imagen positiva de la UE en varios círculos políti-

61 BeEtham, D., op. cit., nota 44.

62 Parliamentary Centre, op. cit., nota 39.

63 Magliveras, K. y Huliaras, A., «Understanding Success and Failure in the Quest for Peace: The Pan-African Parliament and the Amani Forum», en Stavridis, S. y JANCIC, D., op. cit., nota 14, pp. 327342. Véase también, O’Brien, M., Stapenhurst, R. y Johnston, N. (eds.), Parliaments as Peacebuilders in Conflict-affected Countries, Washington DC, World Bank Publications, 2008.

${ }^{64}$ Para más detalles, véanse Redei, L. y Romanyshyn J., «The EU's Invisible Diplomacy: The European Parliament's External Action in the Lead-Up to the Ukraine Crisis», EUSA Biennial Conference, Boston, 2015; Nitiou, C. y Sus, M., «The European Parliament's Diplomacy - a Tool for Projecting EU Power in Times of Crisis? The Case of the Cox-Kwasniewski Mission", Journal of Common Market Studies, vol. 55, 2017, núm. 1, pp. 71-86. Véase también Cox, P., The Ukrainian Crisis and Geopolitics - An Echo from the Past or the Shape of Things to Come?, Dublín, Institute of International and European Affairs, 2014. 
cos y entre la sociedad civil ucraniana. Por supuesto, era una operación de apoyo a la diplomacia tradicional, en este caso tanto de la UE como la de los países UE miembros del grupo de Minsk (que incluye a Francia y Alemania). Y, aunque no pudo ni parar la violencia en el este de Ucrania (Donbass) ni la anexión un año más tarde de Crimea por parte de Rusia, confirma la naturaleza plural de los niveles, actores y funciones de la diplomacia parlamentaria.

\subsection{Tribunas morales}

30. Los parlamentos actúan como tribunas morales en el sistema internacional. Esta es una de las conclusiones que más se menciona en la literatura sobre el tema. Sin entrar en los detalles otra vez, solo se mencionarán dos ejemplos de la diplomacia parlamentaria del PE. El primero de ellos es la defensa por parte del PE de los derechos humanos mediante el Premio Sájarov a la Libertad de Conciencia ${ }^{65}$. Desde 2008 existe también «la Red del Premio Sájarov [que] pone en contacto a los diputados del PE con los galardonados con el premio y la sociedad civil para aumentar la cooperación en materia de derechos humanos que se desarrolla tanto en Bruselas como a nivel internacional». En segundo lugar, el PE ha actuado en los últimos años como un promotor del concepto de Responsabilidad de Proteger (o R2P en sus siglas inglesas), un nuevo concepto internacional que ha revolucionado en cierto punto el mundo con su atención particular a atrocidades como el genocidio, los crímenes de guerra, la limpieza étnica y los crímenes de lesa humanidad. Desde 2005 forma parte del glosario internacional ${ }^{66}$ y fue invocado por el Consejo de Seguridad de la ONU para actuar en el conflicto libio en 2011. Sin tratar del caso en cuestión ${ }^{67}$, es suficiente notar que el PE fue un motor principal para su aplicación por países UE durante la primavera árabe en Libia. Aunque esta actuación, tanto su dimensión de "prevención» como en la de «reconstrucción», no han sido exitosas, hay que señalar que en su dimensión «reacción» sí lo ha sido: porque consiguió parar la masacre de civiles, que es el objetivo primordial de la $\mathrm{R} 2 \mathrm{P}^{68}$.

${ }^{65}$ Un galardón anual creado en 1988 y que lleva el nombre del opositor soviético y físico Andréi Dimitriévich Sájarov (1921-1989), Premio Nobel de la Paz en 1975 e inventor de la bomba de hidrógeno soviética, disponible en $h t t p: / / w w w . e u r o p a r l . e u r o p a . e u / s a k h a r o v p r i z e / e s / h o m e . h t m l$. Véase la lista de los galardonados hasta la fecha en http://www.europarl.europa.eu/sakharovprizeles/laureates.html.

${ }^{66}$ Sobre la Responsabilidad de Proteger, véanse inter alia: BELlAMY, A., Global politics and the Responsibility to Protect. From words to deeds, Londres y Nueva York, Routledge, 2011; Díaz BarRADo, C. M., «La responsabilidad de proteger en el derecho internacional contemporáneo: entre lo conceptual y la práctica internacional», Revista Electrónica de Estudios Internacionales, vol. 24, 2012, pp. 1-40; Thakur, R., The Responsibility to Protect - Norms, laws and the use of force in international politics, Londres y Nueva York, Routledge, 2011.

67 Véase Jeangène Vilmer, J.-B., «Ten Myths About the 2011 Intervention in Libya», The Washington Quarterly, vol. 39, 2016, núm. 2, pp. 23-43.

68 Para detalles véase Stavridis, S. y Fernández Molina, I., «El Parlamento Europeo y el conflicto de Libia (2011): ¿una tribuna moral eficiente?», Revista CIDOB d'Afers Internacionals, vol. 101, 2013, pp. 153-176. 
31. En definitiva, estos dos ejemplos concretos ilustran la actuación como tribunas morales de asambleas parlamentarias. Y los casos comentados anteriormente ponen de manifiesto la realidad de una diplomacia parlamentaria —exitosa o no, porque ni siquiera la diplomacia tradicional siempre obtiene éxitos-.

\section{LAS INSTITUCIONES PARLAMENTARIAS INTERNACIONALES (IPI) COMO DIPLOMACIA PARLAMENTARIA ${ }^{69}$}

32. No sería completo este análisis de la diplomacia parlamentaria si no se mencionaran las IPI ${ }^{70}$, particularmente relevantes para la diplomacia parlamentaria de dos formas diferentes aunque interrelacionadas: primero, el propio establecimiento de una IPI representa una praxis de la diplomacia parlamentaria; segundo, su propio funcionamiento diario, es decir, la diplomacia parlamentaria continúa en y durante las actividades de las IPI.

33. Las IPI son «[i]nstituciones internacionales de carácter parlamentario, cuyos miembros son elegidos o designados por legislaturas o por el electorado para representarlos ${ }^{71}$. Existen entre cuarenta ${ }^{72}$ y alrededor de un centenar ${ }^{73}$. Lo importante es tener en cuenta que su proliferación ocurre en dos fases, una tras la Segunda Guerra Mundial, especialmente en Europa y América Latina, y la segunda después del final de la Guerra Fría a nivel mundial ${ }^{74}$.

34. Existen dos tipos de IPI: una versión más institucionalizada y relacionada formalmente con una organización internacional, y otra más informal y sin vínculo similar ${ }^{75}$. Otras tipologías distinguen entre las IPI de integración

69 Esta Parte del estudio también se basa en la propia experiencia del autor como «observador académico» en las siguientes sesiones de IPI: 2a Sesión ordinaria del Foro Parlamentario Euromediterráneo celebrada en Bruselas en febrero de 2001; 4a Sesión ordinaria de la Asamblea Parlamentaria Euromediterránea (APEM) celebrada en Atenas en marzo de 2008; 31a Sesión Plenaria de la Asamblea Parlamentaria de Cooperación del Mar Negro (PABSEC) celebrada en Atenas en junio de 2008; 3a Sesión Plenaria de la Asamblea Parlamentaria del Mediterráneo (PAM) celebrada en Mónaco en noviembre de 2008; 4a Sesión Plenaria de la Asamblea Parlamentaria Euro-Latinoamericana (EUROLAT) celebrada en Sevilla en mayo de 2010; 21a Sesión Plenaria de la Asamblea Parlamentaria de la Organización para la Seguridad y la Cooperación en Europa (OSCE-PA) celebrada en Mónaco en julio de 2012; 7a Sesión Plenaria de la Asamblea Parlamentaria Euro-Latinoamericana (EUROLAT) celebrada en Atenas en marzo de 2014; 5a Sesión anual de la Conferencia Interparlamentaria sobre la PESC-PCSD celebrada en Roma en noviembre de 2014.

70 Cofelice, A., Parliamentary Institutions in Regional and International Governance: Functions and Powers, Londres, Routledge, 2019.

71 Cofelice, A. y Stavridis, S., «The European Parliament as an International Parliamentary Institution (IPI)», European Foreign Affairs Review, vol. 19, 2014, núm. 2, pp. 145-178, esp. p. 145.

72 De Puig, L. M., International Parliaments, Estrasburgo, Council of Europe Publishing, 2008.

73 Kissling, C., The Legal and Political Status of International Parliamentary Institutions, Berlín, Committee for a Democratic UN, 2011.

${ }^{74}$ Crum, B. y Fossum, J. E., op. cit., nota 19; Costa, O., Dri, C. y Stavridis, S., op. cit., nota 19.

75 Véase SABIC, Z., op. cit., nota 28. Véase también Costa, O., DRI, C. y STAVRIDIS, S., "Conclusions», en Costa, O., DrI, C. y Stavridis, S., op. cit., nota 19, pp. 231-245. 
regional (o interregional) y las demás ${ }^{76}$, es decir, el objetivo de las IPI no es el mismo y como consecuencia se deben analizar de maneras diferentes. Esta tipología es útil porque insiste en las divergencias que existen en las razones de crear las dichas IPI, pero hay que añadir que no toma en consideración otra cuestión importante: las IPI de integración tienen relaciones externas que comprenden las de tipo diplomático parlamentario.

35. Algunas IPI son más activas que otras — con el PE considerado con razón como una de sus expresiones más avanzadas y sofisticadas, incluso en lo que se refiere a sus relaciones internacionales $-{ }^{77} \mathrm{y}$ cubren una amplia gama de políticas. Recientemente, las IPI han tratado de promover la cooperación antiterrorista en todo el mundo. Un buen ejemplo es la cooperación sistemática entre la Asamblea Parlamentaria del Mediterráneo (PAM) y la ONU en ese asunto ${ }^{78}$.

36. Otro tema de investigación relativo a la proliferación de las IPI es si son algo positivo o negativo, y si deberían racionalizarse. Por ejemplo, en el Mediterráneo, hay al menos 23 IPI cuando había solo ocho en 1992. A ellas hay que añadir 66 cámaras de todos los parlamentos nacionales, sin contar las asambleas parlamentarias de los Estados federales o descentralizados en la zona ${ }^{79}$.

\section{CONCLUSIONES}

37. Nuestras conclusiones se refieren principalmente a los ámbitos de la diplomacia parlamentaria que necesitan un análisis más profundo y deben ser objeto de futuras investigaciones.

38. No cabe duda de que la institucionalización de la cooperación interparlamentaria internacional se ha desarrollado mucho más allá de la cooperación «técnica tradicional». Sin embargo, este autor encuentra totalmente improductivo e innecesario un enfoque reciente que sostiene que se debe ignorar la literatura existente sobre diplomacia parlamentaria con el argumento de que se debe priorizar la investigación sobre la cooperación parlamentaria internacional ${ }^{80}$. De hecho, no hay razón para ver contradicción alguna entre ambas temáticas. Lo contrario llevaría a ignorar importantes desarrollos internacionales: por ejemplo, en noviembre de 2018 senadores estadounidenses votaron con una amplia mayoría a favor de frenar el apoyo de la Administración Trump a Arabia Saudí en su guerra en Yémen en par-

\footnotetext{
76 Malamud, A. y Stavridis, S., op. cit., nota 15. Véase también Costa, O., Dri, C. y Stavridis, S., op. cit., nota 19.

77 STAVRIDIS, S. e Irrera, D., op. cit., nota 29.

78 Para detalles véase $w w w . p a m . i n t$.

79 COFELICE, A. y STAVRIDIS, S., Mapping the proliferation of parliamentary actors in the Mediterranean: facilitating or hindering cooperation?, Roma, IAI Working Paper, 2017.

${ }^{80}$ García Chourio, J.-G., "La cooperación parlamentaria internacional en la era de gobernanza global», Revista de Relaciones Internacionales de la UNAM, enero-abril de 2018, núm. 130, pp. 11-51.
} 
te debido al «caso Khashoggi». Si esto fuera analizado desde la perspectiva de «solo un ejemplo más» de cooperación parlamentaria internacional, entonces no sería posible evaluar correctamente la importancia de este proyecto de resolución ${ }^{81}$. Esta falta de aceptación de una nueva realidad parlamentaria global simplemente ignora que existe ya un «mundo de relaciones parlamentarias» ${ }^{82}$, una pluralidad que se debe estudiar como tal.

39. A continuación enunciamos unas preguntas generales sobre el tema para promover posibles pistas para futura investigación: ¿representan todas las actividades internacionales de los parlamentarios «diplomacia parlamentaria»? ¿Cuáles son sus implicaciones para las Relaciones Internacionales, y para la diplomacia como concepto y como práctica? Como señala Miguel Ángel Giménez Martínez — citando a otro practitioner de la diplomacia parlamentaria, Luciano Volante, ex presidente de la Camera dei deputati italiana-, deberán «incorporarse las actividades internacionales [de los parlamentos] en el marco de un proyecto político, en un diseño que defina los agentes, las metas y los plazos, y contribuya a la consolidación del papel internacional de país en forma sinérgica con las políticas gubernamentales» ${ }^{83}$. Es decir, nos queda mucho trabajo para entender, explicar y evaluar este nuevo fenómeno. Hay que avanzar proponiendo una definición de la diplomacia parlamentaria que vaya más allá de unas listas descriptivas, de un catálogo de actividades y acciones internacionales, como tantas producidas hasta la fecha.

40. En cuanto a las líneas en las que deberán concentrarse futuras investigaciones, podemos preguntarnos si la diplomacia parlamentaria debe apoyar, complementar o servir de posible alternativa a la diplomacia tradicional estatal: una cuestión que llega al corazón del mundo moderno. Otra de las cuestiones que debería ser objeto de investigación futura y que ha sido mencionada brevemente en la introducción de este artículo es la relativa a ¿cuál debe ser el rol de los parlamentos en la definición misma del interés nacional (lo que se refiere al dominio «régalien» en francés)?

41. Otro tema importante es el de si se debe hablar con parlamentarios no-democráticos, o interrumpir relaciones con países que defrauden políticamente hablando, interna o externamente. Hay pocas dudas de que la falta de

${ }^{81}$ Véase «Rebuking Trump, senators back effort to suspend U.S. support for Saudi-led war in Yemen», The Washington Post, 28 de noviembre de 2018, disponible en https://www.washingtonpost.com/ powerpost/pompeo-mattis-to-brief-senate-on-saudi-arabia-khashoggi-and-yemen/2018/11/27/ee4e36c0f28a-11e8-bc79-68604ed88993_story.html?utm_term=.a50b74bdade6; véase también PARIS, G., "Affaire Khashoggi: le Sénat inflige un désaveu à Donald Trump - Les sénateurs américains ont envoyé mercredi le signal fort qu'ils souhaitent punir l'Arabie saoudite pour son rôle dans le meurtre du journaliste», $L e$ Monde, 29 de noviembre de 2018.

82 Raube, K., Wouters, J. y MÜftüLer-Bac, M., «A world of parliamentary relations? Parliamentary cooperation and diplomacy in EU external relations», en RAUbE, K., MÜFTÜLER-BAc, M. y Wouters, J. (eds.), Parliamentary Cooperation and Diplomacy in EU External Relations - An Essential Companion, Cheltenham, Edward Elgar Publishing, 2019 (en prensa).

83 Giménez MartíneZ, M.-Á., «Los inicios de la diplomacia parlamentaria en España durante la legislatura constituyente (1977-1979)», Espacio, Tiempo y Forma (Serie V Historia Contemporánea), vol. 24, 2014, pp. 403-415, esp. p. 406. 
democracia en la mayoría de los países involucrados con los cuales se «hace» la diplomacia parlamentaria complica la situación. Pero hay que notar que países no-democráticos también utilizan la diplomacia parlamentaria para promover sus propios intereses, como ocurrió durante la crisis sobre el uso de armas químicas en Siria en 2013: parlamentarios sirios contactaron directamente sus contrapartes francesas con el fin de intentar bloquear cualquier ataque por parte de Francia ${ }^{84}$. Por otras razones esto no ocurrió, pero la causa fue de índole también parlamentario: la Cámara de los Comunes no apoyó la demanda de su gobierno para intervenir militarmente ${ }^{85}$.

42. Otra cuestión interesante es la de averiguar si una proliferación de entes parlamentarios facilita o no, y si consolida o no, la democratización de un continente o de una región como ha sido el caso en Europa desde 1945. No hay que generalizar siempre desde el ejemplo europeo -el PE como la IPI más sofisticada y avanzada en el mundo- ${ }^{86}$ pero lo que era un continente de guerras y conflictos se ha convertido como uno de los más pacíficos y desarrollados tanto políticamente como económicamente. Lo que Shimon Peres ha descrito como: «La UE es un sueño que terminó en un milagro» ${ }^{87}$.

43. También deberá analizarse la relación entre los diferentes niveles de parlamentarismo en el mundo como no solo los parlamentos nacionales o los parlamentos inter/regionales participan en estos procesos, sino también los parlamentos de los entes sub-estatales/autonómicos (las Comunidades Autónomas en España) ${ }^{88}$. Por ejemplo, Cataluña no pertenece a la Organización Internacional de la Francofonía (OIF), pero su Parlament sí que pertenece a la Asamblea Parlamentaria de la OIF. Entre otras cosas, permite una protodiplomacia parlamentaria en un foro internacional donde hay otros miembros que tienen similares políticas e intenciones como Quebec ${ }^{89}$.

${ }^{84}$ StaVridis, S., "The French Parliament and the conflicts in Libya and Syria», en StaVRIDIS, S. y GiANnIOU, M., op. cit., nota 46, pp. 21-41, esp. pp. 35-36.

85 KaARbo, J. y KEnEALY, D., "Precedents, parliaments, and foreign policy: historical analogy in the House of Commons vote on Syria», y Mello, P. A., "Curbing the role prerogative to use military force: the British House of Commons and the conflicts in Libya and Syria», ambos en West European Politics, vol. 40, 2017, pp. 62-79 y 80-100.

86 Véase Cofelice, A. y Stavridis, S., op. cit., nota 79.

87 En un discurso en el PE durante su visita a las instituciones de la UE en marzo de 2013, disponible en http://www.bnaibritheurope.org/bbeurope/en/what-we-do/public-policy/382-shimon-peres-visitseuropean-union-institutions.

${ }_{88}$ Para un estudio jurídico del tema, véase Arrufat CÁrdava, A.-D., Asambleas Autonómicas y Acción Exterior, Valencia, Corts Valencianas, 2009.

89 Véase Palomares Amat, M., La participació del Parlament de Catalunya en l'Assemblea Parlamentària de la Francofonia / La participation du Parlement de Catalogne à l'Assemblée parlementaire de la Francophonie, Barcelona, Parlament de Catalunya, Testimonis Parlamentaris, 37, 2016. Véase también Go, S. (Université Paris 8, France), «Cultural diplomacy at the service of proto-diplomacy? The case of the Generalitat and the Parlament de Catalunya», Paper presented to the 2nd WORKSHOP - Security \& Stability in the Mediterranean and the Middle East organized by the Institute of International Economic Relations (IIER/IDOS) of Athens in collaboration with the Department of Political and Social Sciences of the University of Catania; the Department of Mediterranean Studies of the University of the Aegean (Rhodes); the Université Clermont Auvergne (UCA); Fundación ARAID (Zaragoza); and the Research Unit on European \& International Studies of the University of Zaragoza, with the additional support of the Parliamentary Assembly of the Mediterranean/PAM (Athens, 25-27 de mayo de 2017). 
44. Finalmente, otro tema de estudio debe ser el papel de los individuos, en este caso tanto de los parlamentarios como de los funcionarios trabajando en las secretarías de estos entes parlamentarios. Se necesita más investigación tanto de los informes y otras publicaciones que se producen de manera regular y constante como de las entrevistas sistemáticas sobre estos temas que se ofrecen. Un análisis académico sobre si existe o no "turismo parlamentario» o si solo, como en todas las profesiones y los puestos electos, hay buenas prácticas o malas prácticas. Ejemplo de ello es el caso del senador del Partido Popular, Pedro Agramunt, quien dimitió de su puesto de presidente de la Asamblea Parlamentaria del Consejo de Europa en octubre 2017 cuando se le acusó de beneficiar al gobierno de Azerbaiyán a cambio de sobornos —investigación finalizada en 2018 con la publicación de un informe de más de 250 páginas ${ }^{90}$ que habla de pagos ilegales, de prostitutas o incluso de «caviar»— ${ }^{91}$. Agramunt asegura que todo es falso y que ese informe es inquisitorio ${ }^{92}$.

45. En términos más institucionales cabe preguntarse cómo obtener cierta continuidad en lo internacional; lo que es lo mismo, cómo asegurarse de que los parlamentarios involucrados en diplomacia parlamentaria son también expertos en los temas en los cuales participan. Así, el PE desde hace un tiempo ha armonizado la membresía de sus eurodiputados de la Comisión de Desarrollo con la de su Asamblea Interparlamentaria con los países ACP (África, Caribe y Pacífico). Este sistema permite no solo una mejor coherencia entre el PE y sus relaciones con estos países sino dentro de las diversas opiniones que existen en el mismo $\mathrm{PE}^{93}$.

46. Uno de los objetivos de este estudio es incentivar más investigación sobre la diplomacia parlamentaria. Por el momento, utilizamos la definición siguiente: "Cualquier actividad o acto de carácter diplomático que implica al menos un actor parlamentario (persona o entidad)» ${ }^{94}$. Quizá sería necesario, teniendo en cuenta la opinión de Volante (véase más arriba), añadir «y que intenta impactar sobre un tema internacional o interno con implicaciones internacionales». Caben pocas dudas sobre el hecho de que seguimos en una fase de consolidación del interés académico sobre el tema, pero aún falta mucha investigación, especialmente sobre cómo diseñar criterios de evaluación de su impacto. Por esa razón se necesita mucho trabajo empírico. Este artículo es un primer paso en esta dirección.

\footnotetext{
90 http://assembly.coe.int/Communication/IBAC/IBAC-GIAC-Report-EN.pdf.

91 Sobre la «diplomacia del caviar», véase «Bakou s'achète une influence en Europe», Le Monde, 6 de septiembre de 2017. Véase también SÁNCHEz, Á., "El Consejo de Europa inhabilita 10 años al senador del PP Agramunt por corrupción. No podrá ser observador electoral ni presidir comisiones en una década», El País, 16 de mayo de 2018.

${ }^{92}$ www.lasexta.com, 24 de abril de 2018.

93 Delputte, S., «The ACP-EU Joint Parliamentary Assembly Seen by its Members: Empowering the Voice of People's Representatives?», European Foreign Affairs Review, vol. 17, 2012, núm. 2, pp. 241-260.

94 STAVRIDIS, S., Conferencia sobre «La diplomatie parlementaire dans l'espace méditerranéen: quel impact?», Séminaire régional de l'Organisation Internationale de la Francophonie sur "La diplomatie parlementaire: quel rôle pour les parlements dans les relations internationales?», organizado por la Academia Diplomática de Viena (13-17 de junio de 2016).
} 


\title{
RESUMEN
}

\section{LA DIPLOMACIA PARLAMENTARIA: EL PAPEL DE LOS PARLAMENTOS EN EL MUNDO}

Este texto trata de la diplomacia parlamentaria. Primero pregunta si es un oxímoron o una realidad. Explica cómo el interés ahora también es de tipo académico, después de haber sido inicialmente solo el campo de los practitioners. El estudio muestra cómo, a partir del significado de «diplomacia de la conferencia», el concepto y su práctica se han convertido ahora en una nueva forma de diplomacia. El artículo incluye varios ejemplos de esta práctica. El ensayo también menciona las Instituciones Parlamentarias Internacionales (IPI) como una evidencia empírica de la «parlamentarización» de los asuntos mundiales, pero también de la diplomacia parlamentaria.

Palabras claves: diplomacia parlamentaria, Instituciones Parlamentarias Internacionales (IPI), nuevas formas de diplomacia, parlamentarización, Relaciones Internacionales.

\begin{abstract}
PARLIAMENTARY DIPLOMACY: THE ROLE OF PARLIAMENTS IN THE WORLD

This study is about parliamentary diplomacy. It begins by asking if it is an oxymoron or a reality. It explains how, after having initially been of the realm of practitioners alone, now there is also academic interest in it. The article shows how from its initial meaning of "conference diplomacy», both the concept itself and its practice now represent a new form of diplomacy. The essay includes several illustrations of this practice and also mentions International Parliamentary Institutions (IPIs) as further empirical evidence of the "parliamentarization» of global affairs but also as yet another practical example of parliamentary diplomacy.
\end{abstract}

Keywords: parliamentary diplomacy, International Parliamentary Institutions (IPIs), new forms of diplomacy, parliamentarization, International Relations. 